\title{
Compte-rendu de la séance du 23 novembre 1985
}

La Société Beige de Dermatologie et de Syphiligra-phie s'est réunie au Campus de la Plaine de ГUniversité Libre de Bruxelles, à Гinvitation du Professeur G. Achten.

La matinee est consacrée à la presentation de cas cli-niques en diapositives:

De Maubeuge, J.; Ledoux, M.; Collart, F.; Mullier, J.P.; Vanzeune, J.; Andre, J.; Achten, G.:

Scléromyx-ædème (Arndt-Gottron).

Ledoux, M.; Song, M.; Broeckx, W.; Wanet, J.; Van Maldergem, L.; Goudrain, J.M.; Achten, G.: Pseudo-dermatomyosite et agammaglobulinémie de Bruton.

Gheeraert, P.; Andre, J.; Achten, G.: Sclérædème de Buschke.

Goens, J.; De Maubeuge, J.; Achten, G.: Syndrome Lucky Luke.

Goens, J.; De Maubeuge, J.; Achten, G.: Syndrome de Rosenbloom.

Bernard, C.; Degardin, P.: LE systémique à depart cutané.

De Maubeuge, J.; Mathys, C; Merckx, M.; Andre, J.; Ledoux, M.; Achten, G.; Simonart, J.M.:

Fasceite éosinophile.

Mathys M.; Simonart, J.M.: La capillaroscopie.

Platteau, M.; De Maubeuge, J.; Andre, J.; Achten, G.: Deux cas de chancre mou.

Song, M.; Vincke, G.; Vanachter, H.; Benekens, J.; Achten, G.: Traitement d'une ulcération cutanée à My-cobacterium ulcerans.

Parent, D.; Depre, G.; Pelseneer-Coremans, J.; De-pierreux, M.; Schoutens-Serruys, E.; Heenen, M.: Ar-thrite à Fusarium chez un patient burundais.

De Coninck, A.; Potvin, P.; Van Rensbergen, R.; Willemsen, M.; Roseeuw, D.: Lesions cutanees au cours d'une septicémie à Pseudomonas aeruginosa.

Andre, J.: Lymphadénopathie angio-lymphoblas-tique.

Wanet, J.; Regnier, R.: Réticulose de Crosti.

Dulieu, J.; Parent, D.; Heenen, M.: Mycosis fon-goïde - Essai de traitement par la cyclosporine A.

Ruelens, E.; Feremans, M.; Marcelis, L.; Ardichvili, D.: Pyoderma gangrenosum et leucémie à polynuclé-aires neutrophiles.

Delescluse, J.; Achten, G.: Granulome annulaire profus traité par chlorambucil.

Roseeuw, D.; De Coninck, A.; De Raeve, L.; Willemsen, M.: Adénocarcinome sinuso-nasal.

Pennequin, F.; Dulieu, J.; Parent, D.; Heenen, M.: Sarcome de Kaposi chez un patient soumis à une PU-VAthérapie au long cours.

Laporte, M.; Achten, G.; Gheeraert, P.; Lowy, M.; Vokaer, A.: Léiomyomes multiples.

Germaux, M.A.; Simonart, J.M.; Heimann, R.: Mélanome desmoplastique.

Quevrin, F.; Lowy, M.; Achten, G.: Acanthosis nig-ricans et tumeur rénale.

Al Rustom, K.; Gerard, J.; Pierard, G.E.; Lapière, CM.: Neurocristopathie pigmentogène extrahypophy-saire.

Al Rustom, K.; Pierard, G.E.: Anhidrose régionale sans alteration structurelle de la peau révélant une né-oplasie para-vertébrale.

Nayaert, J.M.; Geerts, M.L.; Kint, A.: Mélanome juvenile: etude immunohistochimique. 
Fierens, F.: Syndrome oculo-digital.

Dinet, Y.; Van Rengen, I.: Maladie de Reiter: un cas sporadique.

Degardin, P.; Simonard, J.P.: Nécrobiose lipoïdique ulcérée cicatrisée par Tassociation persantine-aspirine.

Geeraerts, D.; Lowy, M.; Achten, G.: Pemphigoïde bulleuse traitée par prednisolone et azathioprine.

Van Durme, P.; Lowy, M.: Pemphigus vulgaire.

Willemsen, M.; De Coninck, A.; De Raeve, L,; Roseeuw, D.: Syndrome de Senear-Usher induit par la $\mathrm{Z}>$-pénicillamine.

Decroix, J.; Minet, A.; Eggers, S.: Papillomatose confluente et réticulée de Gougerot-Carteaud. Van Neste, D.; Masmoudi, M.; Mahmound, G,; Leroy, B.; Lachapelle, J.M.: Evalution non invasive des reactions d'irritation: $\Gamma$ exemple des patch-tests au lau-rylsulfate sodique.

Le theme étudié Paprès-midi est le SIDA, avec les communications suivantes:

Achten, G.; Clumeck, N.: SIDA 1985

De Maubeuge, J.; Clumeck, N.: SIDA: Généralités

Andre, J.; Ledoux, M.; De Maubeuge, J.; De Dobbeleer, G.; Schroeder, F.; Cran, S.; Gourdain, J.M.; Clumeck, N.; Achten, G.: Sarcome de Kaposi

Song, M.; Cran, S.; De Maubeuge, J.; Clumeck, N.: Les affections fongiques dans le SIDA

Goens, J.; De Maubeuge, J.; Cran, S.; Ledoux, M.; Deprez, C.; Clumeck, N.; Achten, G.: Les

infections vi-rales dans le SIDA

Tennstedt, D.; Leroy, B.; Minne, G.; Lachapelle, J.M.: A propos de deux cas de SIDA, à manifestations cutanees prémonitoires

Society Proceedings

287

De Maubeuge, J.; Cran, S.; Clumeck, N.: Lesions cutanées et SIDA: varia

Song, M.; Van Malderghem, J.: Reactions cutanées médicamenteuses dans le SIDA

De Dobbeleer, G.; De Maubeuge, J.; Clumeck, N.; Achten, G.: Information et groupes à risques

De Maubeuge, J.; Cran, S.; Clumeck, N.: Prospec-tives thérapeutiques dans le SIDA

Proceedings of the Meeting of March 1,1986

The Belgian Society for Dermatology and Syphilol-ogy met in Leuven in the Department of

Professor H. Degreef.

During the morning the following clinical cases were presented:

A. Dooms-Goossens: Contact allergy to spices in a cook

Snauwaert, J; Van Daele, M.; Drijkoningen, R.: An-giolymphoid hyperplasia with eosinophilia

(Kimura's disease)

Snauwaert, J.: Raynaud's phenomenon and acral sclerosis induced by bleomycin

De Veylder, H.; Acrodermatitis atrophicans chron-ica Pick-Herxheimer

Swinnen, C.: Warty dyskeratoma

Swinnen, C; Willems, L.: Lupus erythematosus -lichen planus overlap syndrome

Swinnen, C: Granulomatous slack skin (granulo-matous chalazoderma)

Biesemans, G.: Neonatal lupus erythematosus

Marien, K.: Erythromelalgia

Dupre, K.: Oral-facial-digital syndrome type I

Dupre, K.; Erven, W.: Hypereosinophilic syndrome

Dupre, K.; Loncke, J.: Keratosis pilaris spinulosa as a paraneoplastic phenomenon 
Vandermaesen, J.; Gladys, K.: Acquired ichthyosis

Maselis, T.; Gladys, K.: CRST syndrome, Sjögren syndrome, and bullous pemphigoid due to Z > -penicilla-mine

Duron, C.: Erythrokeratodermia figurata variabilis Mendes da Costa

Vandermaesen, J.: Hair sinus

Vandermaesen, J.; Van Haute, N.: Focal epithelial hyperplasia

Garmyn, M.; Dewolf-Peeters, C: Juvenile xantho-granuloma ( 2 cases)

Garmyn, M.; Dinet, Y.; Boogaerts, M.: Pyodermia ulcerosa serpiginosa

Borghijs, A.M.: Papulo-necrotic tuberculids of the penis

Borghijs, A.M.; Snauwaert, J.: Non-Hodgkin lym-phoma with morpheiform skin lesions

Debusschere, K.: Proliferating trichilemmal tumor

Debusschere, K.: Multiple basal cell epitheliomata

De Boulle, K.; Drijkoningen, M.; Loncke, J.; Roe-landts, R.: Phytophotodermatitis-like lesions in PUVA patients (5 cases)

De Boulle, K.: Keratosis punctata of the palmar creases

De Boulle, K.: Cryoglobulinemia - Kahler's disease - Plasmoblastic sarcoma

Maselis, T.: Pyogenic granuloma of the nose - Fis-tulisation after infection of an acrylate prosthesis

Maselis, T.: Cowden's disease - Malignant melanoma

The following papers were also presented:

Dooms-Goossens, A.: Airborne contact dermatitis

Lother, Y.; de la Brassinne, M.: Systematic ophthal-mologic examinations during PUVA therapy

Bourlond, A.: LyelГs syndrome due to Vectren (published in this issue, pp. 280-283)

Pierard, G.E.; Pierard-Franchimont, C; Legrain, A.; Darcis, J.M.; Couvreur, Y.; Christophe, J.:

Mollus-cum contagiosum

Darcis, J.M.; Pierard, G.E.: Septicemia and follicu-litis due to Candida albicans in a heroin drug user

The afternoon was devoted to photodermatology, with the following papers:

Roelandts, R.: Historic introduction

Diffey, B.: Factors influencing daily sunlight exposure

De Schryver, F.: Photochemical changes due to UV exposure

Roelandts, R.: Phototoxicity

Vandermaesen, J.; Roelandts, R.: Persistent light sensitivity

van der Leun, J.: Carcinogenic risk of sun exposure

Yong, A.: The use of psoralens in sunscreens; risks versus benefits

Snauwaert, J.; Roelandts, R.; Vlietinck, R.: PUVA therapy of alopecia areata

De Boulle, K.; Roelandts, R.; Stevens, A: Therapy of pain provoked by PUVA.

Elections: Dr. D. Roseeuw was elected full member; Drs L. De Raeve, P. De Selys, D. Dubois, J.L. Michiels, G. Minne, and M. Platteau were elected associate members. 